\title{
CXCL12-CXCR4 SIGNALING PATHWAYS OF ORANGE-SPOTTED GROUPER (EPINEPHELUS COIOIDES) CONTRIBUTE TO NODAVIRUS INFECTION
}

\author{
Chen-Shiou Wu ${ }^{1,2,3}$, Ting-Yu Wang ${ }^{1,2}$, Tzong-Yueh Chen ${ }^{1,2,3,4}$ § \\ ${ }^{1}$ Institute of Biotechnology, National Cheng Kung University, Tainan 70101, Taiwan \\ ${ }^{2}$ Department of Biotechnology and Bioindustry Sciences, National Cheng Kung University, Tainan \\ 70101, Taiwan \\ ${ }^{3}$ Translational Center for Marine Biotechnology, National Cheng Kung University, Tainan 70101, \\ Taiwan \\ ${ }^{4}$ Agriculture Biotechnology Research Center, National Cheng Kung University, Tainan 70101, Taiwan
}

\begin{abstract}
The chemotactic signaling induced by the binding of chemokine CXCL12 to chemokine receptor CXCR4 is of significant biological importance. CXCR4 expression can be detected significantly up-regulated in eye and brain, which are the main targets for nervous necrosis virus (NNV) infection and replication. Understanding of the regulation mechanisms of CXCR4 signaling is essential for revealing its role in physiological and pathological processes. Here we characterized the biological activities of the putative mature peptide of grouper CXCL12, which was expressed in E. coli and purified as a soluble recombinant CXCL12 protein by affinity chromatography. Further investigation of the molecular mechanisms revealed that $\mathrm{CXCL} 12$, the sole ligand for CXCR4, treatment regulated signal transducer and activator of transcription 1 (STAT1), phosphatidylinositol 3-kinase (PI3K)/Akt, protein kinase $\mathrm{C}$ (PKC), and extracellular signal-regulated kinase (ERK) signal pathway and established the existence of pathways that CXCL12/CXCR4 axis in the orange-spotted grouper (Epinephelus coioides). CXCL12 induces the PI3K/Akt activation via CXCR4, and nuclear factor-kappa B (NF- $\mathrm{kB}$ ) p65 is the downstream target of PI3K/Akt signaling pathway. Furthermore, the NNV infected grouper whose expression of p65 increases significantly in brain. In addition, we examined whether CXCL12 influences NNV infection, and assessment of the antiviral activity of CXCL12. These data provide valuable information for further exploration of groupers chemokine signaling pathways and their roles in immune responses to virus infection.
\end{abstract}

\section{KEYWORDS}

CXCL12, CXCR4, nodavirus, Epinephelus coioides, NF-кB

§Corresponding author. Tel.: +886-6-2757575\#65622-610; Fax: +886-6-2766505

E-mail address: ibcty@mail.ncku.edu.tw 